nal in der Gesamtgesellschaft, zu amorph, ihrer selbst nicht bewußt, zu wenig organisiert. M. V. diskutiert sodann die Rolle der Universität einerseits als Markt revolutionärer Ideen, vor allem aber als Reservoir integrationswilliger und sekuritätsbedürftiger "availables“ für jede auftretende politische Führungskraft. Jede artikulierte und halbwegs organisierte Führungsgruppe kann, wie das Beispiel Castros zeigt, in dem durch den Rückzug der alten Oligarchie entstehenden Vakuum erfolgreich sein, wenn man in Rechnung stellt, daß „the desire for power is the central phenomenon" (S. 171). Castros ganze erratische Politik bezwecke, sich unentbehrlich $\mathrm{zu}$ machen und dadurch seine Macht zu festigen. - Auch die Armee komme für diese Rolle in Betracht. Ihre militante Phase der spektakulären, teuren und nutzlosen Waffen sei vorüber; ihr Selbstverständnis werde neu definiert, u. a. als Agent des wirtschaftlichen und sozialen Fortschrittes in der Verantwortung gegenüber dem Gemeinwohl (Beispiel Peru) - wobei allerdings die Armee oft genug beansprucht, alleiniger Interpret dieser Begriffe $\mathrm{zu}$ sein. Selbst eine Art politische Partei, geht sie Koalitionen mit politischen Führern ein, solange diese die Vorrechte der Armee nicht schmälern können. Ein Spannungsverhältnis zur politischen Führung ist unvermeidlich. M. V. diskutiert die Schwierigkeiten, echte Massenparteien $\mathrm{zu}$ organisieren (die auch die Kommunisten erfahren haben).

Seine Schlußfolgerungen - insgesamt nicht sehr originell - zielen dahin, daß, da keine andere dynamische Klasse die ständig expandierenden Funktionen der Gesellschaft wahrnehmen kann, der Staat in die Bresche springen und sie übernehmen muß. Was ist aber der Staat unter den Bedingungen Lateinamerikas? Die Antwort darauf ist M. V.'s Hauptthese: Die angeblich unideologische Ideologie der Manager-Technokraten-Organisatoren. Sie stellen die sich herausbildende neue politische Klasse - Ideologie sei nur Mittel zur Erringung der Macht. Danach interessierten nur noch Mittel und Wege. Die
Analyse der heimischen Situation mache die zahlreichen importierten Modelle und Ideologien überflüssig, die Aktivität der Techniker löse die Zeit der Imitation ab. Diese Aufgabe integriere die Manager-Organisatoren - den tertiären Sektor - nicht nur zur neuen politischen Klasse, sondern damit zugleich zum Staat schlechthin: "what makes it a class in itself in Latin America are its role as an agent of change, its dominant position within the state, and its clearly expressed political will to control the state, to be the state" (S. 194). - Trotz dieser Mischung aus Technokraten-Ideologie und Nationalismus ist die Arbeit für das Selbstverständnis eben des tertiären Sektors wichtig.

Knud Krakau

\section{Henry Wells}

\section{The Modernization of Puerto Rico}

A Political Study of Changing Values and Institutions,

Cambridge Mass., University Press.

Harvard 1969. IX, 440 Seiten

Wenn die Geistesverfassung die vornehmste Bildnerin aller anderen Verfassungen einer Nation ist, dann ist die Vergleichung von Geistesverfassungen die Grundlegung aller Verfassungsvergleichung. Wie schon der Untertitel andeutet, geht es dem Verfasser um die Wandlung eines traditionellen Wertsystems unter dem Einfluß einer Modernisierung, die als Folge der Eroberung von 1898 vor allem als Amerikanisierung sich geltend macht. Dank dieser einmaligen Konstellation hat sich eine Konfrontation der Geistesverfassungen ergeben, wie sie in dieser Unmittelbarkeit und mit solcher Schärfe anderswo nicht vorgekommen ist. Der Verfasser nimmt diese Situation mit Recht zum Anlaß, die streitenden Mentalitäten ausführlich und eindringlich vorzuführen. Das spanische Wertsystem Puerto Ri$\cos$ (21 ff.) ist durch vier Grundzüge bestimmt. Die beiden ersten unter ihnen betreffen die Stellung des Menschen in der Welt: Der Verfasser definiert sie 
als „Fatalismus“ (das Leben wird nicht durch den Menschen, sondern durch eine höhere Macht bestimmt) und als "ascription" (nicht der Mensch wählt seine Stellung in der Gesellschaft, sie ist $\mathrm{ihm}$ vielmehr von einer vorgegebenen Ordnung zugewiesen). Folgerecht erweist sich die Gesellschaft als eine feststehende Hierarchie, die vor allem dem Mann einen höheren Rang zuspricht als der Frau. Angesichts der Vorgegebenheit dieser Ordnung kann der sie durchwaltende Geist nur Ergebenheit („deference") der jeweils Niederen gegenüber den jeweils Höheren sein (26). Die beiden anderen Grundwerte dieses Systems betreffen die Natur des Menschen und interessieren daher hier nicht.

Haltbarkeit und Erträglichkeit dieser fremdgesetzten Ordnung beruhen auf zwei Werten, die der Verfasser unter der Bezeichnung "respect" und "dignidad" beschreibt. "Respect" ist eine wechselseitige Verhaltensweise. Sie wird vor allem auch vom Höheren dem Niederen geschuldet: "The good landowner was one who 'respected' his workers and their families by taking thought for their welfare and by treating them courteously, without condescence" (45/46). Die Würde ist eine allgemeine und gleiche Eigenschaft, die ihrerseits Respekt verlangt. Alle gesellschaftlichen Beziehungen erhalten hierdurch einen menschlich-persönlichen Charakter, wie denn vor allem auch die starke, eindrucksvolle Persönlichkeit als Herrscher einer Institution vorgezogen wird. (33). Derjenige Zug, der nach herkömmlicher Auffassung wahrscheinlich das größere Hindernis der Modernisierung bildet, ist eine unökonomische Gesinnung, die zwar das Geld gewiß nicht verachtet, aber in kommerzieller Geschäftigkeit nicht den höchsten und erst recht nicht den einzigen Sinn des Lebens zu sehen vermag. Noch für die Gegenwart gilt: "For the most part, indeed, leading spokesmen of the two groups have given the impression that welfare values are irrelevant, even ignoble considerations" (242). Daß vor allem das letzte den Amerikanern unbegreiflich, ja verächtlich ist, versteht sich von selbst, zumal wenn man die Schilderung vor Augen hat, die der Verfasser vom amerikanischen Wertsystem gibt (66 ff). Sie verfallen z. B. in geradezu moralische Entrüstung darüber, daß sich die Einwohner nach einem Hurrikan sonnen, anstatt ihre zerstörten Hütten wiederaufzubauen (77).

Der Leser fragt gespannt, ob sich die eigenen Werte gegen Modernisierung und Amerikanisierung behauptet haben. Leider erfährt man hierüber nicht allzuviel, mit Ausnahme etwa einer Andeutung, so wenn (212) die Rede ist von "the continuing importance of traditional Hispanic deference values in Puerto Rican politics". Andererseits heißt es: "Power replaced respect as the focal value" (101), und dem entspricht es, wenn es für die andere Seite heißt: „...f freedom, a 'modern' deference value of the power category". Vor allem aber scheint es die Erziehung zu sein, die die Umwertung der traditionellen Werte befördert (337).

Dieses Buch erschöpft sich nicht etwa in der Beschreibung von Geistesverfassungen. Es geht nicht minder gründlich und wesentlich auf die anderen Verfassungen ein, zwar nicht in systematischer, wohl aber in übersichtlicher historischer Anordnung. Etwa 1940 haben die PuertoRicaner selbst den Entschluß zur Modernisierung gefaßt. Der große Erfolg dieses Entschlusses ist vor allem dem Staatsmann Luis Muñoz Marín und seinen Leuten $\mathrm{zu}$ verdanken. $(136 ; 163$; 189; 293 ff.). Unter ihm und dank der von ihm geschaffenen politischen Stabilität „Puerto Rico was fast becoming a predominantly urban, industrial society." Seit 1953 ist dieses Land kein Treuhandgebiet mehr (248), Bestrebungen zur Herauslösung aus dem Verband mit den Vereinigten Staaten sind ohne Erfolg geblieben: Man hat es um der Wohlfahrt willen vorgezogen, Mitglied dieses großen und nützlichen Wirtschaftsraumes zu bleiben.

Herbert Krüger 